\title{
Apical filling characteristics of carrier-based techniques vs. single cone technique in curved root canals
}

\author{
K. W. Neuhaus ${ }^{1}$ A. Schick $^{1} \cdot$ A. Lussi $^{1}$
}

Received: 12 August 2015 / Accepted: 18 November 2015 / Published online: 27 November 2015

(C) Springer-Verlag Berlin Heidelberg 2015

\begin{abstract}
Objectives In this in vitro study, we assessed filling characteristics (adaptation, homogeneity, sealer percentage, position of the carrier) of warm and cold obturation methods in curved root canals.

Materials and methods A reciprocating method was used to prepare 90 curved roots $\left(25^{\circ}\right.$ average curvature) to an apical size of 25.08 . They were then obturated with either (A) Guttafusion (VDW, Munich, Germany), (B) Thermafil (Maillefer, Ballaigues, Switzerland) or (C) single cone technique using 25.08 gutta-percha (VDW) and sealer (2Seal easymix) ( $n=30$ each group). Five sections in 1-mm steps were obtained from each root, beginning $1 \mathrm{~mm}$ short of the apex. The percentage of voids in contact with root canal walls (PVO), the proportion of voids per area (PVA) and the percentage of sealer per area (PSA) were measured.

Results Little to no differences between Guttafusion and Thermafil were seen in curved root canals with respect to adaptation and homogeneity in the apical region. Both methods showed significantly better adaptation and homogeneity than the single cone technique. The proportion of sealer was significantly greater when roots were obturated with Guttafusion than with Thermafil, but both warm obturation techniques had significantly smaller sealer areas than the single cone technique.

Conclusions Compared to the single cone technique, a more favourable root canal filling with less sealer could be expected from both warm obturation techniques in curved root canals.
\end{abstract}

K. W. Neuhaus

klaus.neuhaus@zmk.unibe.ch

1 Department of Preventive, Restorative and Pediatric Dentistry, University of Bern, Freiburgstrasse 7, 3010 Bern, Switzerland
Clinical relevance The easier-to-handle Guttafusion leads to comparable results as Thermafil in curved root canals.

Keywords Warm vertical obturation - Warm vertical condensation · Centred condensation · GuttaCore . Homogeneity · Adaptation

\section{Introduction}

The prerequisite of good endodontic fillings is to obturate the complex root canal system with gutta-percha and sealer. It is speculated that the dissolution of sealer over time might be responsible for leakage [1], thus promoting endodontic failure. Therefore, good endodontic fillings should bring the obturation material into as close contact as possible to the root canal wall and minimize the sealer content $[2,3]$. Voids in contact with the root canal wall ('interfacial gaps') are potentially problematic, because they can facilitate harbouring of bacteria [4].

Up to now, gutta-percha has been the most suitable obturation material for straight and curved roots, because, particularly after heating, the material becomes viscous and can be condensed to the root canal walls. Earlier studies have shown that root canal systems can be obturated by over $95 \%$ with gutta-percha using different filling techniques [5-9].

Among the cold compaction techniques, lateral condensation is considered as the gold standard [10]. The single cone technique using matching single cones has been described by some authors as being comparable to other root filling techniques [11-15], whereas other studies reported inferior results with this technique $[16,17]$. Less favourable results are due to the geometrical differences between the root canal shape after instrumentation and perfectly round single cone gutta-percha points. Thus, the relative amount of sealer in the single cone 
technique is usually bigger compared to other obturation techniques, because only sealer or entrapped air can fill the space next to the gutta-percha point. A thin layer of sealer between the root canal wall and the gutta-percha should be strived for, because a higher percentage of sealer in a root canal filling might render retreatments more complicated.

Thermafil was introduced as a 'centred' condensation technique that was intended to insert warm thermoplastic guttapercha into a root canal system quickly and predictably [18], resulting in a tight seal [19]. Because the plastic carrier of Thermafil or its location in the root canal might cause some problems during retreatment [20] or during preparation of a post space, a system using cross-linked gutta-percha (GuttaCore, Dentsply, Tulsa, USA) as a carrier material was introduced. Guttafusion and GuttaCore are comparable materials which differ with respect to the modified pincer grip of Guttafusion carrier pins and, more recently, in the colour of the carrier (personal communication, VDW). Therefore, findings reported for GuttaCore are applicable to Guttafusion and vice versa.

While the filling quality in oval-shaped, purportedly straight, single-rooted premolars was found not to be different to warm vertical compaction [21], filling characteristics of thermoplastic gutta-percha with a cross-linked gutta-percha carrier have not yet been examined in curved root canals. The presence of entrapped air/voids in the root filling has been described as its 'homogeneity', while the presence of voids at the root filling/canal wall interface has been described as 'adaptation' [9]. Voids in root canal fillings, especially between gutta-percha and root canal wall, can be regarded as locus minoris resistentiae for bacterial leakage. It seems that root curvature $<25^{\circ}$ might influence the homogeneity and adaptation of root canal fillings [22]. Furthermore, it could be speculated that the gutta-percha carrier of Guttafusion becomes softened after heating and thus might lack sufficient pressure to bring down gutta-percha in a curved root canal.

Therefore, we wanted to compare the homogeneity, adaptation and the percentage of sealer in the apical portion of root canal fillings of curved root canals obturated with Guttafusion/Thermafil and the single cone technique. As a secondary outcome, we were interested in the location of the carrier of the two warm compaction methods.

\section{Materials and methods}

\section{Tooth selection}

We chose and prepared 90 single palatal roots from a pool of non-treated extracted human molars and premolars. Exclusion criteria were apical resorptions, caries, cracks, open apex or missing patency with a $\# 10$ needle. The roots were placed in distilled water until needed.

\section{Working length and curvature measurement}

Working length was established using a $\# 10 \mathrm{~K}$-file, which was inserted into the root canal until the tip of the instrument was just visible. Working length was defined to be $0.5 \mathrm{~mm}$ shorter than this length. All roots were then radiographed in two perpendicular planes with a $\# 15 \mathrm{~K}$-file inserted. The visible curvatures of the roots were measured three times using ImageJ software (NIH, Bethesda, MD, USA). After calculating the arithmetic mean, the higher of the two angles defined the curvature of the root. We included only roots with an angle $>15^{\circ}$ [23]. The roots were then negotiated using a 25.08 reciprocating instrument (Reciproc R25, VDW, Munich, Germany) according to the manufacturer's instructions. Sodium hypochlorite $3 \%$ was used as intermittent irrigation solution. The final rinsing protocol consisted of $5 \mathrm{ml}$ of $3 \%$ sodium hypochlorite and $3 \times 20$ s $17 \%$ EDTA solution with passive ultrasonic activation using non-cutting irrigation needles (Irrisafe, VDW).

\section{Root canal filling/test groups}

The roots were stratified according to their curvature angles and then divided evenly into three groups: (A) Guttafusion (VDW), mean curvature $25.8^{\circ}\left( \pm 10.8^{\circ}\right)$; (B) Thermafil (Maillefer, Ballaigues, Switzerland), mean curvature $25.0^{\circ}$ $\left( \pm 9.2^{\circ}\right)$ and $(\mathrm{C})$ single cone (VDW), mean curvature $24.6^{\circ}$ $\left( \pm 8.9^{\circ}\right)$. In groups $A$ and $B$, working length and maintenance of the apical constriction were verified using the respective verifier. The roots were dried with paper points. Epoxy resin sealer (2Seal easymix, VDW) was put into the root canals using coated R25 paper points (VDW). For groups A and B, excess sealer was removed using dry paper points until they were speckled with sealer material. In these groups, the obturators were preheated (Thermaprep, Maillefer) and then inserted slowly into the root canal up to working length. They were maintained in place for $30 \mathrm{~s}$. While the cores in group A could easily be twisted off, the cores in group B were removed using a high-speed, $40-\mu \mathrm{m}$ diamond. In group $\mathrm{C}$, the gutta-percha cone R25 (VDW) was rolled in the sealer and then inserted straight into the root canal avoiding pumping movements. The roots were placed in distilled water at $37^{\circ} \mathrm{C}$ until required.

\section{Specimen preparation}

After $48 \mathrm{~h}$ setting time, the roots were sawn horizontally at $120 \mathrm{rpm}$ (Bühler IsoMet low speed saw, IL, USA) under constant water cooling. The saw blade had a thickness of $0.35 \mathrm{~mm}$, one section took about $30-40 \mathrm{~s}$ to saw. Six parallel horizontal sections with 1-mm distance between each cut were made beginning $1 \mathrm{~mm}$ short of the apex. Thus, five specimens were obtained and mounted on a glass plate. The surfaces 
were polished using 2400 grit paper $(8 \mu \mathrm{m})$ in a polishing machine (Struers LaboPol-21, Willich, Germany) after which they were placed in an ultrasonic bath for another $3 \mathrm{~min}(\mathrm{O}$. Kleiner AG, Wohlen, Switzerland). The specimens were then bench-dried and photographed with a digital stereo microscope at $\times 32$ magnification (Leica M420, Heerbrugg, Switzerland).

\section{Outcome parameters}

The pictures were magnified, measured and analysed using specialized software (LAS v3.7, Leica). One examiner (AS) carried out all measurements. The circumference and the area of root canal filling material (gutta-percha, sealer, carrier); circumference and area of voids; and area of sealer were measured.

The outcome parameter used to assess adaptation of the filling for each section was the proportion of voids in contact with the root canal walls compared to the whole outline (PVO). The proportion of filling in contact with the root canal walls (PFO) was calculated as 1 - PVO. The outcome parameter used to assess homogeneity of the filling for each section was the proportion of voids per area (PVA). Furthermore, we calculated the proportion of sealer in the whole area (PSA) of root canal filling in each section. Finally, we determined whether or not the carriers were in contact with the root canal wall. The following scores were assigned: $0=$ no core, $1=$ wall contact and $2=$ centred position.

\section{Statistics}

Statistics were generated using R software (v. 2.15.1, www.rproject.org, Vienna) and the extensions 'nparLD' and 'exactRankTests'. Because the variables of proportion of voids per area (PVA), PVO and percentage of sealer per area (PSA) were not normally distributed, nonparametric ANOVAs for repeated measurements were performed [24].

The first step was to analyse each section independently. If the nonparametric ANOVA showed global significant impacts, further subtests (subANOVAs) were performed for every filling material to determine whether the layers had a different impact on the outcome values. Finally, post hoc exact Wilcoxon rank-sum tests were performed to detect differences in medians between the three filling materials for every layer. Owing to the high number of sub- and post hoc tests, no correction for multiple testing was applied.

In the second step, median values of the five sections of each root were calculated, and statistical analysis was also done for the comparisons of mPVA, mPVO and mPSA. The Kruskal-Wallis rank-sum test was applied for the global tests, and the Wilcoxon test was applied for post hoc tests, if necessary. For this analysis, a Holm correction for multiple testing was applied.
The overall final position of the carriers in groups A and B (either touching the wall or in the centre) was compared using the exact Fisher test with a $2 \times 2$ contingency table. Thereby, a tooth was only considered in the analysis when position of the carrier was clear, i.e., when out of five sections, the majority corresponded to one of the following codes: $0=$ no carrier, $1=$ carrier central and $2=$ carrier in contact with the wall. Ten teeth were thus excluded because the position of the carrier was unclear. The level of significance was set at 0.05 .

\section{Results}

During mechanical negotiation of the root canals, no instrument was separated.

\section{Adaptation (PVO)}

Nonparametric ANOVA showed that there was a global significant interaction $(p=0.015)$ between filling and layer. Further subtest ANOVA showed a statistically significant impact of the layers on all three filling materials (Guttafusion $p=0.036$; Thermafil $p=0.028$; single cone $p=0.03$ ). The adaptation of the two warm compaction methods (groups $\mathrm{A}$ and B) increased towards the apex, while in group $\mathrm{C}$, it became decreased. However, significant differences were found only in the apical section between $\mathrm{A} / \mathrm{B}$ and $\mathrm{C}(p=0.01$ and 0.03 , respectively) (Table 1$)$. No global differences were detected for the median adaptation values (mPVO) (Fig. 1).

\section{Homogeneity (PVA)}

Nonparametric ANOVA showed that there was a global significant interaction $(p=0.011)$ between filling and layer. Further subtest ANOVA showed a significant impact of the layers on group $\mathrm{C}$ only (single cone: $p=0.015$ ). The apical layer in the single cone group had significantly more voids than the other layers (Table 1). Within the Guttafusion group, the apical layer also showed the largest amount of voids and the largest $95 \% \mathrm{CI}$, although this difference was not found to be significant. Hence, the global comparison of homogeneity (mPVA) showed a significant difference between Thermafil and single cone only ( $p=0.009$; Fig. 2$)$.

\section{Sealer percentage (PSA)}

Nonparametric ANOVA showed that there is a global significant interaction $(p<0.003)$ between filling and layer. Further subtest ANOVA showed a significant impact of the layers on all groups $(p<0.001)$. The relative amount of sealer decreased from apex upwards in the warm filling techniques (groups A and B), whereas with the single cone technique, there was generally more sealer detectable in all sectioned layers. 
Table 1 Means $(95 \% \mathrm{CI})$ of the filling characteristics (\%) according to each layer

\begin{tabular}{|c|c|c|c|c|c|c|c|c|c|}
\hline \multirow[b]{2}{*}{$\begin{array}{l}\text { Distance from } \\
\text { apex }(\mathrm{mm})\end{array}$} & \multicolumn{3}{|l|}{ Guttafusion } & \multicolumn{3}{|l|}{ Thermafil } & \multicolumn{3}{|l|}{ Single cone } \\
\hline & PFO & PVA & PSA & PFO & PVA & PSA & $\mathrm{PFO}$ & PVA & PSA \\
\hline 1 & $97.7(95.7-99.7)$ & $2.1(0-5.5)$ & $29.7(20.8-38.7)$ & $97.6(95.3-99.9)$ & $1.1(0-2.3)$ & $27.9(18.2-37.6)$ & $95.4(93-97.8)$ & $3.2(1.1-5.2)$ & $43.2(35.2-51.1)$ \\
\hline 2 & $98(96-100)$ & $1.3(0.2-2.3)$ & $14.6(10.8-18.4)$ & $97.4(93.7-100)$ & $1.1(0-2.7)$ & $13.4(7.1-19.7)$ & $98.1(96.7-99.6)$ & $1.1(0.3-1.9)$ & $33.6(27.9-39.4)$ \\
\hline 3 & $96.7(94.2-99.2)$ & $0.6(0.2-1)$ & $7.5(6-8.9)$ & 97.8 (96.1-99.6) & $0.4(0-1)$ & $6.8(5.1-8.3)$ & $97.4(95.1-99.7)$ & $1(0.2-2.9)$ & $33.9(28.3-39.5)$ \\
\hline 4 & $96.2(93.5-98.9)$ & $0.8(0.3-1.3)$ & $7.4(5.8-8.9)$ & 97.6 (95.9-99.3) & $0.5(0.1-0.9)$ & $5.6(4.4-6.8)$ & $98.5(97.4-99.5)$ & $0.5(0.2-0.8)$ & $37.8(32.4-43.3)$ \\
\hline 5 & $94.7(91.2-98.3)$ & $1.2(0-2.6)$ & $7.3(6-8.6)$ & 95.7 (92.7-98.6) & $0.8(0.1-1.5)$ & $6(4.9-7.1)$ & $97.1(94.8-99.4)$ & $1(0.3-1.8)$ & $40.8(35.8-45.8)$ \\
\hline Mean & $96.7(95.5-97.8)$ & $1.2(0.6-1.8)$ & $13.3(10.9-15.7)$ & $97.2(96.1-98.3)$ & $0.8(0.4-1.2)$ & $11.9(9.3-14.6)$ & $97.3(96.4-98.2)$ & $1.4(0.8-1.8)$ & $37.8(35.2-40.5)$ \\
\hline
\end{tabular}

PFO proportion of filling in contact with root canal outline, $P V A$ proportion of voids per area, $P S A$ proportion of sealer per area

Comparison of sealer percentage was different in all groups (Fig. 3).

\section{Position of the carriers}

After excluding 10 roots with unclear carrier positions, we analysed 50 roots to determine the final position of the carriers inside the root canal filling. The frequency of these positions (wall/centre) was 9/17 in group A (Guttafusion) and 20/4 in group B (Thermafil). This difference was highly significant $(p<0.001)$.

\section{Discussion}

Regarding the adaptation of gutta-percha to the root canal wall, Guttafusion and Thermafil led to significantly denser root canal fillings than the single cone technique. The filled areas in the apical $5 \mathrm{~mm}$ largely corresponded to the values

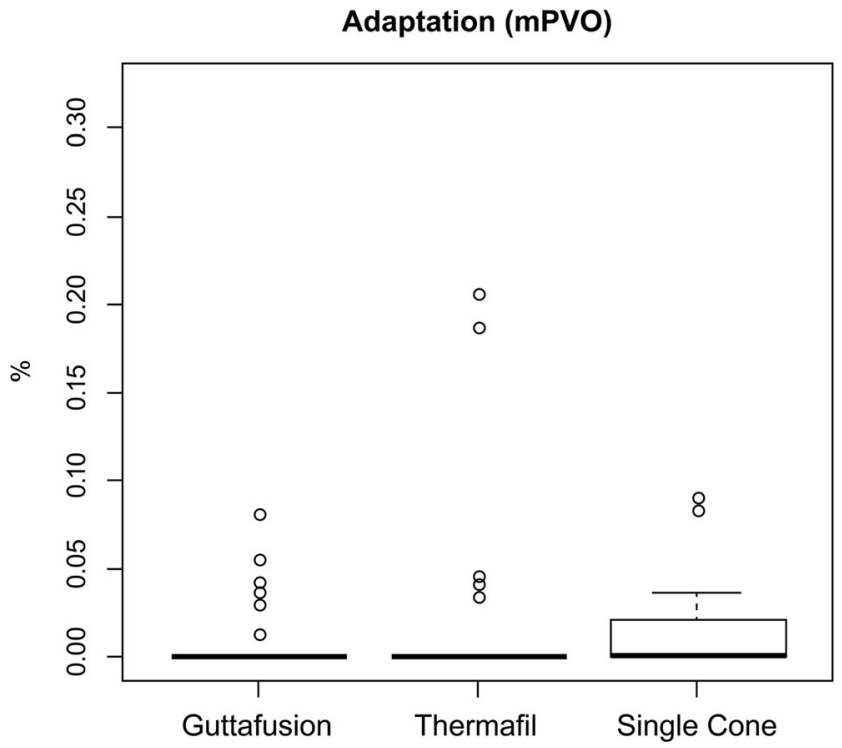

Fig. 1 No global differences were detected with respect to adaptation of filling material (median proportion of voids in contact with the wall; mPVO) described in the literature. ElAyouti et al. [9] found that for the Thermafil method, $91.3 \%(88.9-93.6 \%)$ of the root canal filling was in contact with the wall. However, in our study, the mean value was $97.2 \%(96.1-98.3 \%)$ for Thermafil and $96.7 \%$ (95.5-97.8 \%) for Guttafusion, respectively. Assuming that non-overlapping $95 \%$ confidence intervals represent statistically significant differences, the warm root canal fillings in our study were significantly better adapted than those in the study by ElAyouti et al. The same applies to the findings for PVA; ElAyouti et al. reported mean values of $4 \%(2.6-5.3 \%)$ whereas we found $0.8 \%(0.4-1.2 \%)$ and $1.2 \%(0.6-1.8 \%)$ for Thermafil and Guttafusion, respectively. A closer look at the results reported by ElAyouti et al. reveals that the first (apical) section was responsible for the difference in the mean values. While their findings for sections 2-5 are within the range of our findings, their first section is clearly an outlier. One possible explanation for this finding could be that ElAyouti et al. prepared the roots with an apical enlargement up to \#45-60 with K-files. That means the most apical portion of the roots had a taper of 0.02 while the more

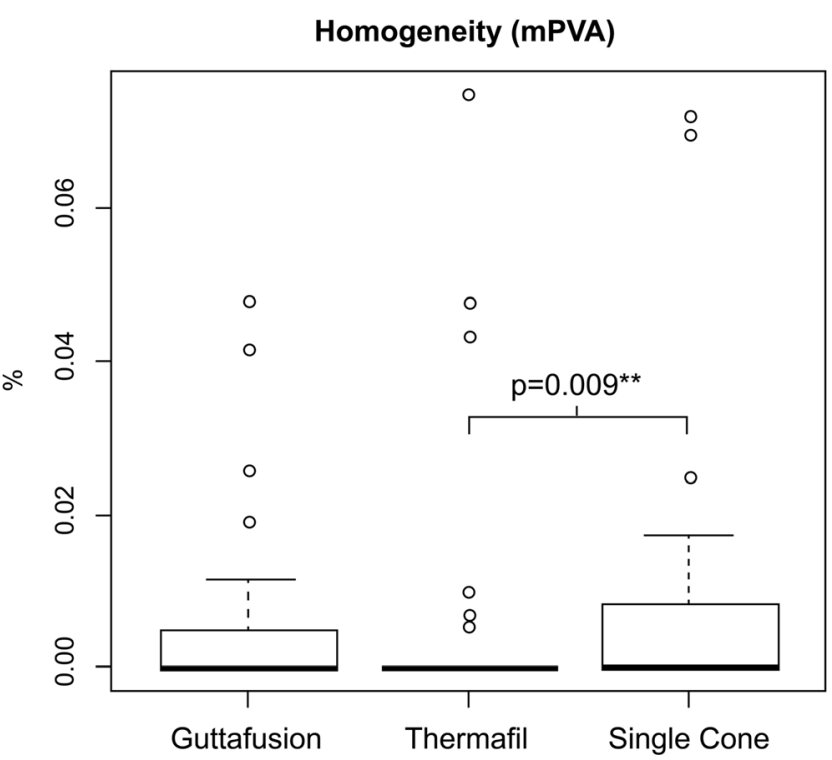

Fig. 2 There was a significant difference with respect to filling homogeneity as indicated by median proportion of voids per area (mPVA) 
Sealer percentage (mPSA)

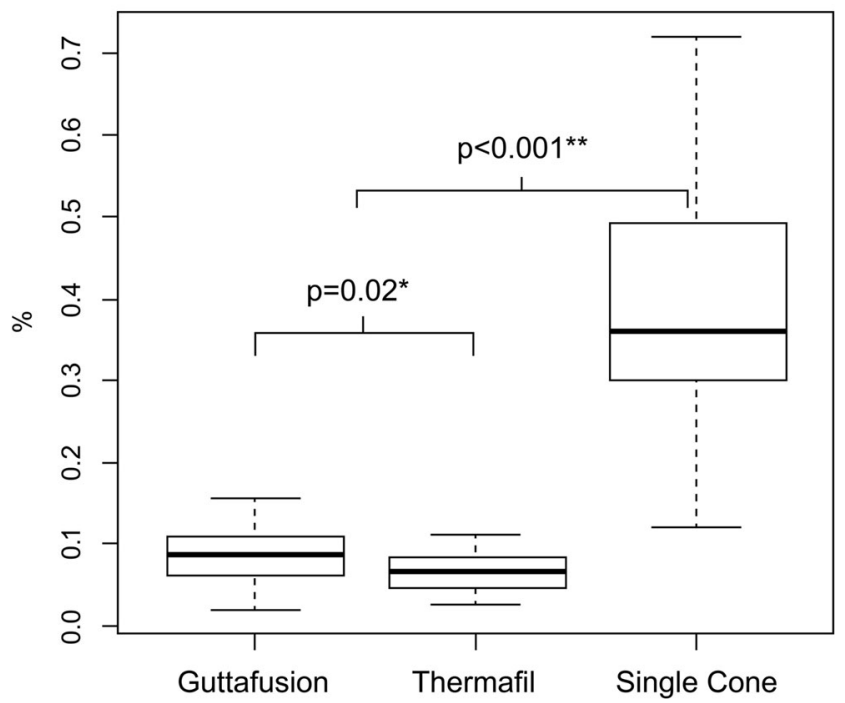

Fig. 3 Comparison of median sealer percentages (mPSA) showed significant differences

coronal portions had a greater taper. We prepared the roots with an R25 instrument, i.e., with a 25.08 taper. We assume that with a constant taper, the conditions for achieving a welladapted obturation with Thermafil and Guttafusion might be more favourable than an enlarged apical box preparation. Previous research has shown that using Thermafil, even with an apical preparation size of 60 but respecting a continuous taper, the percentage of gutta-percha in the apical $2-4 \mathrm{~mm}$ is $>97 \%$ [6].

In a recently published study, the adaptation of GuttaCore and Thermafil examined oval-shaped root canals using micro-computed tomography $(\mu \mathrm{CT})$ and compared to cold lateral condensation [21]. No difference between the two warm compaction methods was found, and the percentage of voids was reported to be between 1 and $2 \%$ in the apical $4 \mathrm{~mm}$, which is in line with our findings. However, oval root canals were included in that study, and no information was provided regarding curvature or the roots. Furthermore, only 10 teeth per group were included in the latter study. We included 30 teeth per group, which results in a greater statistical power.

We chose curved root canals because the published studies on GuttaCore/Guttafusion have so far not examined the impact of root canal curvature on the filling characteristics. It seems that for root curvatures $>25^{\circ}$, the difference between cold and warm techniques becomes significant [22]. The average curvatures of our roots were exactly in that range (Table 1). Generally, the advantage of filling quality of both warm vertical techniques over the single cone technique could be demonstrated. But comparing Guttafusion and Thermafil, it could be uncertain whether in curved root canals enough pressure can be maintained to fully bring the material down to the apex because the carrier of Guttafusion is cross-linked guttapercha and not plastic material. We were able to show that root canal curvature does not negatively influence the quality of warm vertical obturation with a guttapercha-based carrier, because in terms of homogeneity and adaptation, there was no difference to fillings with Thermafil. However, we noted a slightly larger portion of sealer in Guttafusion fillings $(13.3 \%)$ compared to Thermafil $(11.9 \%)$, which was found to be statistically significant. Whether or not this difference is clinically meaningful remains a matter of speculation. Gulsahi et al. found a percentage of sealer in the apical $4 \mathrm{~mm}$ of $0.12 \%$ using Thermafil [25]. By contrast, the sections of the single cone group always showed a sealer proportion of $20 \%$ per section examined. These large-scale areas of sealer probably represent a weak point of a root filling because multiple voids in a single section were observed only in the presence of a high percentage of sealer. The goal of bringing the gutta-percha as close to the root canal wall as possible, and thus counteracting microleakage [26], was clearly not achieved by the single cone technique.

However, it has to be kept in mind that the percentage of sealer in the apical section might be different under in vivo conditions as a result of tissue pressure from the periapical region. It has been reported that the sealer area using Thermafil could be reduced to about $1.2 \%$, when 'firm apical pressure' was used to bring Thermafil obturators to working length [8], but excess material was not examined in that study.

Another finding of this study is the location of the carrier inside the root canal. We detected a significant difference between Guttafusion and Thermafil, the Guttafusion carrier being located in two thirds of the specimens in the middle of the root filling, while the Thermafil touched the walls in more than $80 \%$ of the specimens. These results are caused by the rigidity of the plastic carrier of Thermafil and also demonstrate that the Guttafusion carrier flexibly adapts to the anatomy of curved root canals. This could have an influence on revision, while in root canals obturated with Thermafil, the removal of the plastic carrier remains a challenge [27], depending on whether or not the operator can detect the revision groove of the carrier, the Guttafusion should give a constant tactile resistance upon removal. Furthermore, because the centrally positioned carrier of Guttafusion cannot be melted by heat, the use of hot pluggers during retreatment of Guttafusion has a limited effect. However, in terms of effectiveness, Guttafusion/GuttaCore seems to be removed faster than Thermafil [20].

Although we used 'cone-fitting' gutta-percha (R25, 25.08 taper) from the same producer for the single cone technique, it 
was clear that large areas remained that were filled by sealer only or that contained voids. Even if this technique might be less time-consuming than warm obturation or cold lateral condensation [28], dentists should be aware that root canal shape is far from being round in the apical region when an instrumentation of 25.08 taper is performed. Especially when watersoluble sealers are used, the filling quality might deteriorate quickly when the single cone technique is applied. 'Cone-fit' does not in the least equal 'perfect fit'.

We measured voids in sections of the apical third. Clinically, voids could be problematic when they are in contact with root canal walls, as they could harbour bacteria and represent a source of leakage [4]. Earlier leakage studies tested the sealing quality of root canal filling. However, leakage studies may not reliably measure what they should measure [29] and they are prone to misinterpretation depending on the model used [29, 30]. Leakage of root canal filling could also be tested with a suitable microbial model. However, it was shown that the classical two-chamber model for measuring bacterial penetration is also error-prone [31]. Microtomographic images are nowadays recommended to measure voids in root canal fillings, because it is a non-destructive method [21]. However, depending on the sealer used, and depending on the chosen parameters, discrimination between gutta-percha and sealer might be difficult in $\mu \mathrm{CT}$ images. Histology therefore still seems to be a viable method to assess the quality of root canal fillings.

\section{Conclusion}

In this laboratory study, we found little to no differences in the quality of the warm obturation techniques with Guttafusion and Thermafil in curved root canals with respect to adaptation and homogeneity in the apical region. Clinicians should be aware that - compared to the carrierbased techniques Guttafusion or Thermafil - the proportion of sealer is much greater when roots are obturated with single cone technique. Compared to Thermafil, the single cone technique showed three times and Guttafusion two times bigger proportion of voids per area at the apical section. Whether or not this finding is clinically meaningful warrants further investigation.

Acknowledgments The help of Lukas Martig (Institute of Mathematical Statistics and Actuarial Science, University of Bern) with statistics is hereby gratefully acknowledged. The materials used in this study were donated by VDW and Maillefer.

Compliance with ethical standards Ethical approval

This article does not contain any studies with human participants or animals performed by any of the authors.

Conflict of interest The authors declare that they have no competing interests.

\section{References}

1. Wu MK, Wesselink PR (1993) Endodontic leakage studies reconsidered. Part I. Methodology, application and relevance. Int Endod J 26:37-43

2. Kontakiotis EG, Wu MK, Wesselink PR (1997) Effect of sealer thickness on long-term sealing ability: a 2-year follow-up study. Int Endod J 30:307-312

3. Sakaue H, Komatsu K, Yoshioka T, Ishimura H, Ebihara A, Suda H (2013) Evaluation of coronal leakage and pathway of dye leakage after obturation with various materials for open apical foramina. Dent Mater J 32:130-137

4. Orstavik D, Nordahl I, Tibballs JE (2001) Dimensional change following setting of root canal sealer materials. Dent Mater 17: 512-519

5. Dummer PM, Kelly T, Meghji A, Sheikh I, Vanitchai JT (1993) An in vitro study of the quality of root fillings in teeth obturated by lateral condensation of gutta-percha or Thermafil obturators. Int Endod J 26:99-105

6. Jarrett IS, Marx D, Covey D, Karmazin M, Lavin M, Gound T (2004) Percentage of canals filled in apical cross sections - an in vitro study of seven obturation techniques. Int Endod J 37: 392-398. doi:10.1111/j.1365-2591.2004.00821.x

7. De-Deus G, Gurgel-Filho ED, Magalhaes KM, Coutinho-Filho T (2006) A laboratory analysis of gutta-percha-filled area obtained using thermafil, system B and lateral condensation. Int Endod J 39:378-383. doi:10.1111/j.1365-2591.2006.01082.x

8. De-Deus G, Maniglia-Ferreira CM, Gurgel-Filho ED, Paciornik S, Machado AC, Coutinho-Filho T (2007) Comparison of the percentage of gutta-percha-filled area obtained by Thermafil and System B. Aust Endod J 33:55-61. doi:10.1111/j.1747-4477.2007.00047.x

9. ElAyouti A, Kiefner P, Hecker H, Chu A, Lost C, Weiger R (2009) Homogeneity and adaptation of endodontic fillings in root canals with enlarged apical preparation. Oral Surg Oral Med Oral Pathol Oral Radiol Endod 108:e141-e146. doi:10.1016/j.tripleo.2009.04.022

10. Peng L, Ye L, Tan H, Zhou X (2007) Outcome of root canal obturation by warm gutta-percha versus cold lateral condensation: a metaanalysis. J Endod 33:106-109. doi:10.1016/j.joen.2006.09.010

11. Whitworth J (2005) Methods of filling root canals: principles and practices. Endod Topics 33:2-24

12. Horsted-Bindslev P, Andersen MA, Jensen MF, Nilsson JH, Wenzel A (2007) Quality of molar root canal fillings performed with the lateral compaction and the single-cone technique. J Endod 33:468471. doi:10.1016/j.joen.2006.12.016

13. Souza EM, Wu MK, van der Sluis LW, Leonardo RT, Bonetti-Filho I, Wesselink PR (2009) Effect of filling technique and root canal area on the percentage of gutta-percha in laterally compacted root fillings. Int Endod J 42:719-726. doi:10.1111/j.1365-2591.2009. 01575.x

14. Wu MK, Bud MG, Wesselink PR (2009) The quality of single cone and laterally compacted gutta-percha fillings in small and curved root canals as evidenced by bidirectional radiographs and fluid transport measurements. Oral Surg Oral Med Oral Pathol Oral Radiol Endod 108:946-951. doi:10.1016/j.tripleo.2009.07.046

15. Schafer E, Nelius B, Burklein S (2012) A comparative evaluation of gutta-percha filled areas in curved root canals obturated with different techniques. Clin Oral Investig 16:225-230. doi:10.1007/ s00784-011-0509-Z

16. Marciano MA, Ordinola-Zapata R, Cunha TV, Duarte MA, Cavenago BC, Garcia RB, Bramante CM, Bernardineli N, Moraes IG (2011) Analysis of four gutta-percha techniques used to fill mesial root canals of mandibular molars. Int Endod J 44:321329. doi:10.1111/j.1365-2591.2010.01832.x

17. Somma F, Cretella G, Carotenuto M, Pecci R, Bedini R, De Biasi M, Angerame D (2011) Quality of thermoplasticized and single 
point root fillings assessed by micro-computed tomography. Int Endod J 44:362-369. doi:10.1111/j.1365-2591.2010.01840.x

18. Johnson WB (1978) A new gutta-percha technique. J Endod 4:184 188. doi:10.1016/S0099-2399(78)80173-3

19. Lares C, elDeeb ME (1990) The sealing ability of the Thermafil obturation technique. J Endod 16:474-479. doi:10.1016/S00992399(07)80176-2

20. Beasley RT, Williamson AE, Justman BC, Qian F (2013) Time required to remove guttacore, thermafil plus, and thermoplasticized gutta-percha from moderately curved root canals with protaper files. J Endod 39:125-128. doi:10.1016/j.joen.2012.10.014

21. Li GH, Niu LN, Selem LC, Eid AA, Bergeron BE, Chen JH, Pashley DH, Tay FR (2014) Quality of obturation achieved by an endodontic core-carrier system with crosslinked gutta-percha carrier in single-rooted canals. J Dent 42:1124-1134. doi:10.1016/j. jdent.2014.04.008

22. Leung SF, Gulabivala K (1994) An in-vitro evaluation of the influence of canal curvature on the sealing ability of Thermafil. Int Endod J 27:190-196

23. Schneider SW (1971) A comparison of canal preparations in straight and curved root canals. Oral Surg Oral Med Oral Pathol 32:271-275

24. Brunner E, Domhof S, Langer F (2002) Nonparametric analysis of longitudinal data in factorial experiments. Wiley, New York

25. Gulsahi K, Cehreli ZC, Kuraner T, Dagli FT (2007) Sealer area associated with cold lateral condensation of gutta-percha and warm coated carrier filling systems in canals prepared with various rotary NiTi systems. Int Endod J 40:275-281. doi:10.1111/j.1365-2591. 2006.01213.x

26. Keles A, Ahmetoglu F, Ocak MS, Dayi B, Bozkurt A, Orucoglu H (2014) Comparative analysis of three different filling techniques and the effects of experimental internal resorptive cavities on apical microleakage. Eur J Dent 8:32-37. doi:10.4103/1305-7456.126237

27. Hayakawa T, Tomita F, Okiji T (2010) Influence of the diameter and taper of root canals on the removal efficiency of thermafil plus plastic carriers using ProTaper retreatment files. J Endod 36:16761678. doi:10.1016/j.joen.2010.06.013

28. Gound TG, Sather JP, Kong TS, Makkawy HA, Marx DB (2009) Graduating dental students' ability to produce quality root canal fillings using single- or multiple-cone obturation techniques. J Dent Educ 73:696-705

29. Souza EM, Pappen FG, Shemesh H, Bonanato-Estrela C, BonettiFilho I (2009) Reliability of assessing dye penetration along root canal fillings using methylene blue. Aust Endod J 35:158-163. doi: 10.1111/j.1747-4477.2009.00161.x

30. Camps J, Pashley D (2003) Reliability of the dye penetration studies. J Endod 29:592-594. doi:10.1097/00004770-20030900000012

31. Rechenberg DK, De-Deus G, Zehnder M (2011) Potential systematic error in laboratory experiments on microbial leakage through filled root canals: review of published articles. Int Endod J 44:183194. doi:10.1111/j.1365-2591.2010.01821.x 